\title{
Validez y confiabilidad del instrumento de vímculo parental en madres de familia de la ciudad de Toluca
}

Validity and reliability of parental attachment instrument for mothers of the Toluca city

\author{
Erika Robles Estrada \\ Hans Oudhof van Barneveld \\ Susana Silvia Zarza Villegas \\ Lourdes Gabriela Villafaña Montiel
}

\section{Resumen}

La presente investigación tuvo como objetivo validar y confiabilizar el Instrumento de Vínculo Parental de Parker, Tupling y Brown (1979), en una muestra de 125 mujeres mexicanas de la ciudad de Toluca. El instrumento original está constituido por dos escalas; sobreprotección y cuidado con 25 reactivos que permiten obtener los estilos vinculares: control sin afecto, constricción afectiva, vínculo ausente y vínculo óptimo. Se aplicó de manera individual y colectiva en su vivienda o lugar de trabajo de las participantes. Para llevar a cabo la validez se utilizó el análisis factorial y para obtener la confiabilidad el Alpha de Cronbach. De acuerdo con los resultados, quedaron 22 reactivos finales que permiten explicar el $56 \%$ de la varianza con una consistencia interna Alpha de Cronbach de 0.93. Por otra parte, se mantuvieron las escalas Sobreprotección con una confiabilidad de Alpha de Cronbach de 0.96 y Cuidado con 0.70. Se concluye que el Instrumento de Vínculo Parental es válido y tiene una consistencia interna adecuada, además cabe mencionar que la mayoría se ubicó en, o tuvo tendencia a un tipo de vínculo saludable como promedio u óptimo, en contraste con una minoría que se caracterizó por un vínculo control sin afecto, constricción afectiva, ausente o débil.

\section{Abstract}

The purpose of the present research was to validate and to establish the reliability of the Parental Attachment Instrument developed by Parker, Tupling \& Brown (1979), in a Mexican sample consisting of 125 women from the city of Toluca. The original instrument is constituted by two scales, overprotection and care, with a total of 25 items which measure attachment styles: control without affection, affective constriction, absent attachment and optimal attachment. The instrument was administered individually and in small groups at home or at the workplace of the participants. Factor analysis and Cronbach's Alfa were calculated in order to assess its validity and reliability. According to the results, 22 final items were retained, accounting for $56 \%$ of the total variance and with an internal consistency (Cronbach's Alpha) of 0.93. Both scales of the original instrument were maintained: Overprotection and Caretaking, with values for Cronbach's Alpha being 0.96 and 0.70, respectively. It is concluded that the instrument has adequate validity and reliability for Mexican samples. Additionally, the results indicate that the majority of the participants presented a tendency towards healthful attachment (optimal or average), although a minority was characterized by a more negative attachment type of control without affection, affective constriction and weak or absent bonds..

Palabras clave: Vínculo parental, madres, familia, validez, confiabilidad, estilos vinculares.

Keywords: Parental attachment, mothers, family, validity, reliability, attachment styles. 


\section{introducción}

El estudio sobre la relación entre el niño y la madre se ha convertido en el centro de un gran número de investigaciones. Las aportaciones de Bowlby con la teoría del vínculo en la década de los cuarenta a los setenta se centraron en los efectos que el vínculo tiene en los niños como elemento esencial para el desarrollo de una personalidad saludable en la vida adulta, de ahí el interés de validar un instrumento que permitiera la evaluación psicológica del vínculo en adultos.

De acuerdo con la teoría de Bowlby (2000), existe una tendencia natural de los seres humanos a establecer relaciones íntimas y estables con personas determinadas, la cual comienza desde los primeros meses de vida y se evidencia en las diversas formas que el niño tiene para obtener y mantener la proximidad con una figura significativa. Así se establece la ligadura Madre-Hijo, la cual es el resultado de un conjunto de pautas de conducta características, en parte preprogramadas, que se desarrollan en el entorno corriente y que tienen el efecto de mantener al niño en una relación estrecha con la figura materna, a la cual se le denomina vínculo, que es la ligadura emocional característica del mundo mental, en el que se distinguen tres áreas: intrasubjetiva o intrapsíquica, intersubjetiva o interpersonal y transubjetiva o que trasciende a lo social (Bernstein, citado por Lartigue, 1994). Este primer contacto dará origen al tipo de vínculo el cual, según Bartholomew (1997), se desarrolla tempranamente y se mantendrá generalmente durante toda la vida

De esta manera surge la conducta vincular, que Bowlby (2000) identifica como cualquier tipo de conducta que tiene como resultado el logro o la conservación de la proximidad con otro individuo claramente identificado al que se considera mejor capacitado para enfrentar al mundo. La actividad que realizan los padres para permitir esta conducta se denomina conducta de atención, que tiene que ver con la satisfacción de las necesidades del hijo. Al res- pecto Melis et al. (2001) señala que la interrelación establecida entre la conducta vincular en el niño con la conducta de atención por parte de los cuidadores determinará el tipo y la calidad del vínculo, en el cual influyen factores como la experiencia individual, la constitución genética y las influencias culturales (Weaver y De Waal, 2002).

La teoría enfatiza la importancia de que el niño se sienta capaz de depender de sus figuras de apego y también en la habilidad de estas figuras para contener y proteger al niño, especialmente en momentos de mayor necesidad. El sistema comportamental del apego ha sido hipotetizado como fundamental para la sobrevivencia del niño, pues busca las condiciones de seguridad con sus cuidadores, especialmente bajo las condiciones de amenaza (Martínez y Pía, 2005). Esto tiene que ver con lo que Bowlby (1969) identificó como modelo operativo interno, que se define como una representación mental de sí mismo y de las relaciones con los otros, y que hace posible anticipar, interpretar y responder a la conducta de sus figuras de apego, ya que integra experiencias presentes y pasadas en esquemas cognitivos y emocionales.

Bowlby (1998) desde sus inicios propuso un modelo del desarrollo con claras implicaciones para la psicopatología, ya que el estilo vincular refleja la interacción entre la personalidad del niño, la familia y el entorno social más amplio, de ahí la importancia que el vínculo se encuentre sentado en bases firmes, pues de lo contrario puede dar origen a diversos trastornos en la personalidad. De hecho, mucha de la psicopatología que hoy en día se observa, en niños y adultos, está relacionada con la problemática del abandono espiritual, social y emocional de los niños que puede dar origen a la delincuencia, depresión, psicopatía, trastornos psicosomáticos, entre otros (Yárnoz, 2003).

En este sentido Bucchheim, Brisch y Kächele (1998) afirman que los problemas de vinculación a menudo se transmiten transgene- 
racionalmente, a menos que alguien rompa la cadena. Al respecto Main, Kaplan y Cassidy (1985) consideran que las representaciones mentales de los padres acerca de sus experiencias infantiles de apego determinan el patrón que sus hijos establecen con ellos, lo cual implica cierta continuidad a través de las generaciones.

Ainsworth, colega de Bowlby en 1978 y precursora de la teoría del vínculo, fue la primera que propuso la medición de relaciones vinculares con el argumento de que las relaciones madre-hijo diferían de unos a otros, pero que además estas se podían clasificar. También postuló que la conducta de la madre en los primeros meses de vida del niño es un buen predictor del tipo de relación entre ambos (Ainsworth, 1985). Ainsworth, Blehar, Waters y Wall (1978) propusieron el método para evaluar los diferentes estilos de apego en niños de 12 a 18 meses, basado en las reacciones del niño ante una serie de separaciones y reuniones con su madre y un extraño amistoso, llamando a esto la "Técnica de la Situación Extraña" Ilevada a cabo en un laboratorio diseñado como salón de juegos. La valoración se hizo tomando en cuenta el grado de incomodidad, la aflicción que experimenta el niño y principalmente las estrategias que utiliza para enfrentar sus necesidades de proximidad. A partir de los resultados, al niño se le clasifica en alguno de los tres estilos de apego: seguro, inseguro-evitante y ambivalente-resistente. A estos se agregó otro más, llamado por Main y Salomon (1990) como "vínculo desorganizado o desorientado", puesto que los sujetos no mostraban un patrón claro durante la técnica de la situación extraña.

Estas primeras mediciones de los estilos vinculares se centraron principalmente en niños. Posteriormente se inició la medición en adultos por diversos autores como Parker, Tupling y Brown (1979), quienes diseñaron un instrumento (el que aquí se valida) centrado en los recuerdos que tanto hombres como mujeres adultos poseían de su madre durante la infancia hasta la edad de 16 años, constituido por dos escalas (cuidado y sobreprotección) que en combinación dan diferentes estilos vinculares que son:

1. Vínculo óptimo: Son aquellos padres que obtienen puntajes altos en la escala de cuidado y bajos en la de sobreprotección. Se caracterizan por ser afectuosos, empáticos y contenedores emocionalmente y, a su vez, favorecen la independencia y la autonomía.

2. Vínculo ausente o débil: Los padres que obtienen puntajes bajos en cuidado y bajos en sobreprotección. Se caracterizan por presentar frialdad emotiva, indiferencia y negligencia; al mismo tiempo son padres que favorecen la independencia y la autonomía.

3. Constricción afectiva: Son aquellos padres que puntúan con alto puntaje de cuidado y alto puntaje en sobreprotección. Se caracterizan por presentar afectuosidad, contención emocional, empatía y cercanía, por un lado, y al mismo tiempo son controladores, intrusivos, tienen un contacto excesivo, infantilizan y previenen la conducta autónoma de sus hijos.

4. Control sin afecto: Son aquellos padres que puntúan con bajo cuidado y alta sobreprotección. Se caracterizan por presentar frialdad emotiva, indiferencia y negligencia, al mismo tiempo que son controladores, intrusivos, tienen un contacto excesivo, infantilizan y previenen la conducta autónoma.

5. Promedio: Son aquellos padres que obtienen puntajes promedio en ambas escalas.

Aunque se han realizado diversos estudios sobre el vínculo con las figuras parentales, hasta ahora se conoce poco sobre instrumentos de origen mexicano que midan este constructo, 0 en su caso que se hayan validado en muestras mexicanas. Ante esto surge la necesidad de validar y llevar a cabo la confiabilidad del Instrumento de Vínculo Parental en una muestra de mujeres de la ciudad de Toluca, con la finalidad de que contribuya en el acervo de la medición en psicología en México y que pueda ser utilizado para investigaciones posteriores en el medio cultural mexicano. 


\section{Método}

\section{Participantes}

Los participantes fueron 125 mujeres de la ciudad de Toluca, Estado de México, con edades entre 25 y 50 años con diferente nivel educativo en igual proporción. La muestra fue de tipo intencional y se obtuvo de acuerdo con el número de reactivos del instrumento, tomando como criterio cinco personas por cada uno de ellos. El criterio de inclusión fue que las participantes hubieran vivido con su madre hasta los 16 años.

\section{Instrumento}

El Instrumento que se validó es el de Vínculo Parental de Parker, Tupling y Brown (1979), que mide la percepción de la conducta y actitud de los padres en relación con el sujeto en su infancia y adolescencia, hasta los 16 años, es decir una especie de historia de las relaciones vinculares. Es un instrumento tipo Likert con opciones de respuesta que van de 1 (siempre) a 4 (nunca), constituido por dos escalas representadas en 25 reactivos: 1. Cuidado (12 ítems) que se refiere por un lado a la afectuosidad, contención emocional, empatía y cercanía, y por otro, la frialdad emotiva, indiferencia, negligencia; y 2. Sobreprotección (13 ítems) que apunta al control, sobreprotección, intrusión, contacto excesivo, infantilización y prevención de la conducta autónoma.

Cada escala puede ser utilizada de manera independiente o conjunta, obteniendo así un puntaje para cuidado y otro para sobreprotección. El obtener puntajes de ambas escalas permite elaborar cinco tipos de vínculos parentales: control sin afecto, constricción afectiva, vínculo ausente, vínculo óptimo y vínculo promedio.

\section{Procedimiento}

Los datos se recolectaron en una colonia de la ciudad de Toluca y se aplicó el instrumento de manera individual en la vivienda o lugar de trabajo de las participantes, con previa autorización de ellas y garantizando la confidencialidad de la información.

\section{Análisis de datos}

Para obtener la validez y confiabilidad del Instrumento de Vínculo Parental se utilizó el análisis de factores, que sirve para determinar el número y la naturaleza de un grupo de constructos subyacentes en un conjunto de mediciones, mientras que para la consistencia interna del instrumento se empleó el Alpha de Cronbach.

\section{Resultados}

De acuerdo con los resultados obtenidos en el análisis factorial con rotación varimax del Instrumento de Vínculo Parental, se encontraron 2 factores con valores Eigen o autovalores mayores a uno, a partir de dos criterios: el punto de quiebre de la varianza y la claridad conceptual. Los factores seleccionados contienen 22 reactivos finales que permiten explicar el 56\% de la varianza con una consistencia interna Alfa de Cronbach de .93. Asimismo, se mantuvieron las dos escalas originales del instrumento Sobreprotección y Cuidado; la primera con una confiabilidad de Alpha de Cronbach de .96 y la segunda con .70, como se puede observar en la Tabla 1.

Los 2 factores resultantes abarcaron 22 reactivos, mismos que constituyeron el diseño final del instrumento, quedando de la siguiente manera como se puede ver en la Tabla 2.

\section{Tabla 1}

Varianza del Análisis Factorial del Instrumento de Vínculo Parental

\begin{tabular}{llccccc}
\hline $\begin{array}{c}\text { Número } \\
\text { de factor }\end{array}$ & $\begin{array}{c}\text { Nombre } \\
\text { del factor }\end{array}$ & $\begin{array}{c}\text { Número de } \\
\text { reactivos }\end{array}$ & $\begin{array}{c}\text { Valores } \\
\text { Eigen }\end{array}$ & $\begin{array}{c}\text { Porcentaje } \\
\text { de la } \\
\text { Varianza }\end{array}$ & $\begin{array}{c}\text { Porcentaje } \\
\text { de la varianza } \\
\text { acumulada }\end{array}$ & $\begin{array}{c}\text { Alpha } \\
\text { de Cronbach }\end{array}$ \\
\hline 1 & Sobreprotección & 11 & 5.27 & 21.08 & 21.08 & .96 \\
2 & Cuidado & 11 & 2.97 & 11.89 & 32.97 & .70 \\
\hline
\end{tabular}


Tabla 2

Análisis Factorial y Alpha de Cronbach del Instrumento de Vínculo Parental

\begin{tabular}{|c|c|c|}
\hline & $\begin{array}{l}\text { Factor } 1 \\
\text { Sobreprotección }\end{array}$ & $\begin{array}{l}\text { Factor } 2 \\
\text { Cuidado }\end{array}$ \\
\hline $\begin{array}{l}\text { Sentía que no podía cuidar de mí mismo (a), a menos que } \\
\text { él estuviera cerca. }\end{array}$ & .914 & \\
\hline Era sobre protector (a) conmigo & .888 & \\
\hline Trataba de hacerme dependiente de ella & .859 & \\
\hline Me permitía vestirme como se me antojara & .843 & \\
\hline Me dejaba salir todo lo que yo quería & .837 & \\
\hline Me daba toda la libertad que yo quería & .814 & \\
\hline Invadía mi privacidad & .746 & \\
\hline Le gustaba que tomara mis propias decisiones & .704 & \\
\hline Trataba de controlar todo lo que yo hacía & .700 & \\
\hline No quería que creciera & .691 & \\
\hline Evitaba que yo saliera solo (a) & .641 & \\
\hline $\begin{array}{l}\text { No me elogiaba } \\
\text { No conversaba mucho conmigo } \\
\text { Me hacía sentir que no era deseado (a) } \\
\text { No parecía entender lo que yo quería o necesitaba } \\
\text { Era afectuosa conmigo } \\
\text { Me regaloneaba } \\
\text { Parecía entender mis problemas y preocupaciones } \\
\text { Se entretenía conversando conmigo } \\
\text { Me hablaba con voz amistosa y cálida } \\
\text { Me sonreía frecuentemente } \\
\text { Tenía la capacidad de reconfortarme cuando me sentía } \\
\text { molesto (a) o perturbado (a) }\end{array}$ & & $\begin{array}{l}.880 \\
.823 \\
.822 \\
.790 \\
.776 \\
.747 \\
.717 \\
.592 \\
.588 \\
.574 \\
.546\end{array}$ \\
\hline Alpha Total & .93 & \\
\hline
\end{tabular}

De acuerdo con los puntajes obtenidos por las participantes en cada una de las escalas del instrumento (sobreprotección y cuidado) se encontró que el $68.8 \%$ de la muestra total se encuentra en algún tipo de vínculo, siendo el más alto el Vínculo Promedio con el $33.6 \%$ y el más bajo el Vínculo Ausente o Débil con 3.2\% (Ver tabla 3). 


\section{Tabla 3}

Distribución de Frecuencias de acuerdo con el tipo de Vínculo Parental

\begin{tabular}{lcc}
\hline Tipo de vínculo & Frecuencia & Porcentaje \\
\hline Promedio & 42 & $33.6 \%$ \\
Óptimo & 21 & $16.8 \%$ \\
Control sin afecto & 10 & $8.0 \%$ \\
Constricción afectiva & 9 & $7.2 \%$ \\
Ausente o débil & 4 & $3.2 \%$ \\
Total & $\mathbf{8 6}$ & $\mathbf{6 8 . 8} \%$ \\
\hline
\end{tabular}

El $30.5 \%$ restante de la muestra total no se definió completamente en ningún tipo de vínculo, no obstante, en la tabla 4 se muestran las tendencias arrojadas de acuerdo con los puntajes obtenidos. Se encontró el porcentaje más alto con un 14 por ciento en el tipo "Constricción afectiva".
Es preciso mencionar que se llevaron a cabo las comparaciones del tipo de vínculo por edad, escolaridad y horas de trabajo de las participantes, no encontrando diferencias estadísticamente significativas.

\section{Tabla 4}

Distribución de Frecuencias de acuerdo a las tendencias con los Vínculos Parentales

\begin{tabular}{lcc}
\hline Tipo de vínculo & Frecuencia & Porcentaje \\
\hline Promedio & 7 & $5.6 \%$ \\
Óptimo & 3 & $2.4 \%$ \\
Control sin afecto & 10 & $8.0 \%$ \\
Constricción afectiva & 18 & $14.4 \%$ \\
Ausente o débil & 1 & 0.8 \\
Total & 39 & $\mathbf{3 1 . 2 \%}$ \\
\hline
\end{tabular}




\section{Discusión}

El Instrumento de Vínculo Parental de Parker, Tupling y Brown (1979), validado para muestras mexicanas, contempla dos escalas que permiten clasificar diferentes estilos vinculares: sobreprotección y cuidado, que se basan en el recuerdo que se tiene de la madre hasta los 16 años. De tal manera que estas escalas tienen que ver con representaciones mentales o "modelos operantes" como los llamó Bowlby (1986) y tienen la capacidad de integrar experiencias pasadas y presentes (con el cuidador primario) como también esquemas cognitivos y emocionales relacionados con tales experiencias. La propuesta de Bowlby en relación a los modelos operantes internos es uno de los conceptos más relevantes de su legado, en la medida que forma la base desde la cual las personas inician y mantienen otras relaciones cercanas. Además, serían relativamente estables a lo largo del ciclo vital (Bretherton, 1999; Fronaguy, 1999). Este concepto es clave en la medición del apego adulto. Por lo tanto, los resultados muestran que las dos escalas obtenidas mantienen esta relación entre la experiencia pasada y el estilo vincular en la edad adulta, puesto que se basan en el recuerdo. Los estilos vinculares obtenidos se mantuvieron también de acuerdo con la escala original y son: vínculo óptimo que como su nombre lo indica es el ideal y tiene que ver con el apoyo; vínculo ausente o débil, caracterizado por el rechazo parental; constricción afectiva, identificado por sobreprotección; control sin afecto, donde se da la negligencia del cuidador primario pero además se ejerce control excesivo; y vínculo promedio, que son aquellos padres que obtienen puntajes promedio en ambas escalas.

Cabe mencionar que el instrumento fue adaptado en Chile por Albalá y Sepúlveda (citado por Martínez y Pía 2005) en 1997 y se obtuvieron buenos niveles de confiabilidad de acuerdo con el Alpha de Cronbach (sobreprotección .93, cuidado .78) similares a los obtenidos en México con variaciones mínimas (sobreprotección .96, cuidado .70), con la diferencia de que en Chile se replicaron los 25 reactivos y en Mé- xico fueron eliminados tres de ellos, quedando 22. De manera general se ha encontrado que este instrumento ha sido utilizado ampliamente a nivel mundial (Manassis, Owens, Adam, West \& Sheldon-Keller, 1999; Mancini, D'Olimpio, Prunetti, Didonna \& Del Genio, 2000; Cheng \& Furnham, 2004; Favaretto, Torresani \& Zimmerman, 2001; Myhr, Sookman \& Pinard, 2004; Willinger, Diendorfer-Radner, Willnauer, Jorgl \& Hager, 2005).

Esta clasificación guarda estrecha relación con el modelo de Ainsworth (1985) de los estilos vinculares, se puede decir que el vínculo óptimo y el promedio que se manifiesta cuando la madre se caracteriza por ser afectuosa y empática con los hijos, favorecedora de la independencia y la autonomía (Parker, Tupling y Brown, 1979), guardando similitudes con el tipo de apego seguro en donde la madre es accesible, receptiva y cálida (Ainsworth, 1985). Mientras que el vínculo ausente o débil, referido como aquél que caracteriza a la madre como fría, emotiva e indiferente, tiene que ver con el apego resistente definido por una madre que muestra disponibilidad en ciertas ocasiones pero en otras no, y es indiferente mostrando las amenazas de abandono como medio de control (Bowlby, 1989).

En cuanto al tipo de vínculo constricción afectiva, los creadores de instrumento de esta investigación, Parker et al. (1979), señalan que la madre tiene como característica el ser afectuosa por un lado y al mismo tiempo controladora e intrusiva. De acuerdo con el estudio de Ainsworth (1985), este vínculo sería el equivalente al tipo de apego desorganizado o desorientado, que no permita la autonomía y donde hay control hacia el pequeño.

Finalmente, el vínculo control sin afecto, el cual es generado por una madre que presenta frialdad e indiferencia (Parker et al., 1979), se compara con el apego evitante de Ainsworth (1985), en el cual el niño supone que cuando busque cuidados no recibirá atención o cariño sino rechazo, por ello intentará volverse autosuficiente para vivir sin el apoyo de otros. 
Cabe mencionar que de la muestra total la mayoría se ubicó en algún estilo vincular de acuerdo con los puntajes obtenidos en las escalas sobreprotección y cuidado, pero no se permitió la identificación del estilo en casi una tercera parte de las participantes, aunque se pudo detectar una tendencia hacia el estilo de constricción afectiva.

En este sentido, se plantea la posibilidad de desarrollar una nueva versión del instrumento con otras dimensiones válidas y confiables que permitan la identificación de estilos vinculares en toda la muestra, y que se pueda utilizar en diversos contextos para detectar las relaciones en riesgo de la diada Madre-Hijo, puesto que el vínculo entre ambos es básico en el concepto de calidad en las relaciones tempranas, tal y como fue expuesto por Bowlby (1988), representando un modelo de desarrollo humano con claras implicaciones para la salud y la patología. Los fuertes lazos creados durante la infancia entre el niño y su figura de apego no sólo ayudan a la supervivencia de la especie, sino al individuo durante los años de mayor vulnerabilidad. La evaluación constante de la figura vincular como alguien que puede proporcionar una base segura en caso de necesidad, así como del individuo mismo como alguien merecedor o no de cuidados, le da a la relación entre madre e hijo una importante función organizacional en la dinámica de las personas.

La ventaja del instrumento validado aquí es que permite determinar, de acuerdo con la obtención de las normas, un estilo vincular relativamente rápido. No obstante, es pertinente mencionar que no en todos los casos se llegó a una clasificación precisa, ya que en algunos de ellos sólo se arrojaron las tendencias a cierto tipo de estilo. Asimismo, al realizarse la validación únicamente en una muestra de mujeres, quedó fuera la medición en varones y en niños. De tal manera que se considera necesario vislumbrar en una futura investigación la creación de instrumentos de medición del apego en adultos tanto mujeres como en hombres, así como en niños, lo que permitirá hacer un análisis más fino y detallado de los datos, al mismo tiempo que fortalezca la medición del vínculo en el contexto mexicano.

Por último, esta investigación se centró en la obtención de la validez y confiabilidad del Instrumento de Vínculo Parental y en la descripción de los tipos vinculares de la madres participantes, sin embargo, sería interesante indagar posteriormente la influencia que tiene el desarrollo y la salud mental de la propia madre, como un factor desencadenante de rasgos similares en el propio bebé; y cómo el desempeño de estos rasgos le permitan funcionar como mamá. 


\section{Referencias}

Ainsworth, M, (1985). Attachment across the life span. Bulletin of the New York Academy of Medicine, 61, 792-812.

Ainsworth, M., Blehar, M. Waters, E. \& Wall, S. (1979). Patterns of Attachment Assed in the strange situation and at home. New Jersey: Lawrence Earlbaum.

Bartholomew, K. (1997). Adult attachment processes: individual and couple perspectives. British Journal of Medical Psychology. 70, 249-63.

Bowlby, J. (1969/1989). El apego y la pérdida-2. Barcelona: Paidós.

Bowlby, J. (1998). El apego y la pérdida-1. Barcelona: Paidós.

Bowlby, J. (2000). El apego y la pérdida-3. Barcelona: Paidós.

Bowlby, J. (2001) Una base segura. Aplicaciones clínicas de una teoría del apego. Barcelona: Paidós.

Bretherton, I. (2000). Des modalites aux modeles internes: La perspective de theorie de l' attachment. En O. Halfon, F. Anserment \& B. Pierrehumbert (eds.), Filiations psychiques (pp.102-115). Paris: PUF

Bucchheim, A., Brisch, K. H. y Kächele, H. (1998). The differential relevance of attachment representation, emotion-abstraction patterns, and narrative style: A computer- based text analysis of the Adult Attachment Interview. Psychotherapy Research, 10, 390-407.

Cheng, H. \& Furnham, A. (2004). Perceived parental rearing style, selfesteem and self criticism as predictors of happiness. Journal of Happiness Studies, 5, 1-21

Favaretto, E. Torresani, S. \& Zimmerman, C. (2001). Further results on the reliability of the parental bonding instrument in an Italian sample of schizophrenic patients and their parents. Journal of Clinical Psychology, 57(1), 119-129.

Fonagy, P. (1999). Psychoanalysis and attachment theory. En J. Cassidy \& P. R. Shaver (Eds.). Handbook of attachment: Theory, research and clinical applications (pp. 595-624). New York: Guilford Press.

Lartigue, T. (1994). Guía para la observación del Vínculo Materno-Infantil durante el primer año de vida. México: Universidad lberoamericana.

Lartigue, T. Y Vives, A. (1992). Apego y vínculo. Comunicación preliminar. Cuadernos de Psicoanálisis, XXV (1-2), 45-53.

Main, M. \& Salomon, J. (1990), Procedures for identifying infants as disorganized/disoriented during Ainsworth Strange Situation. En M. Greenberg, D. Cicchetti \& M. Cummings (Eds.). Attachment in the preschool years: Theory research and intervention (pp. 121-160). Chicago: University of Chicago Press.
Main, M., Kaplan, N. \& Cassidy, J. (1985). Security in infancy, childhood and adulthood: A move to the level of representation. En I. Bretherton \& E. Waters (Eds.). Growing points of attachment theory and research. Monographs of the Society for Research in Child Development, 50. Serial no. 209, (pp. 66-104). Chicago: University of Chicago Press.

Manassis, K., Owens, M., Adam, K. S., West, M. \& Sheldon-Keller, A. E. (1999). Assessing attachment: Convergent validity of the Adult Attachment Interview and the Parental Bonding Instrument. Australian and New Zealand Journal of Psychiatry, 33(4), 559-567.

Mancini, F., D'Olimpio, F., Prunetti, E., Didonna, F. \& Del Genio, M. (2000). Parental Bonding: can obsessive symptoms and general distress be predicted by perceived rearing practices? Clinical Psychology and Psychotherapy, 7, 201-208.

Martínez, C. y Pía, M. (2005). La evaluación del apego en el adulto: Una revisión. Revista Psykhe, 14(1), 181-191.

Melis, F., Dávila, M. Ormeño, V., Vera, V., Greppi, C. y Gloger, S. (2001). Estandarización del PBI (Parental Bonding Instrument), versión adaptada a la población entre 16 y 64 años del Gran Santiago. Revista Chilena de Neuropsiquiatría, 30, 132-139.

Myhr, G., Sookman, D. \& Pinard, G. (2004). Attachment security and parental bonding in adults with obsessive compulsive disorder compared to depressed outpatients and healthy controls. Acta Psychiatrica Scandinavica, 109, 447-456.

Parker, G. Tupling, H. \& Brown, L. (1979). A parental Bonding Instrument. British Journal of Medical Psychology, 52, 1-10.

Weaver, A. y De Waall, F. (2002). An index of relationship quality based on attachment theory. Journal of Comparative and Physiological Psychology. 116, 93-106.

Willinger, U., Diendorfer-Radner, G., Willnauer, R.Jorgl \& Hager, V. (2005). Parenting stress and parental bonding. Behavioral/Medicine, 31(2), 63-69.

Yárnoz, S. (2003). Normalidad y patología: aportaciones de la teoría del apego. Recuperado el 12 de mayo de 2008, R de http://psiquiatría. com/interpsiquis2003/9767. 